\title{
Introduction to the Internet of Things: Providing Services Using Smart Devices, Wearables, and Quantified Self Minitrack
}

\author{
Fred Riggins \\ North Dakota State University \\ fred.riggins@ndsu.edu
}

\author{
Tayfun Keskin \\ University of Washington Bothell \\ keskin@uw.edu
}

This minitrack addresses issues organizations face as they seek to provide services to end-users through wearable or autonomous mobile devices using the emerging Internet of Things (IoT) platform. The IoT allows connectivity of billions of mobile devices that can perform physical, sensing, and analytical services to users. Many of these devices exist in physical proximity of the user making up part of the user's personal area network (PAN). Others are wearable devices that sense and track metrics about the quantified self and make up the user's body area network (BAN). Finally, other mobile devices are semi-autonomous or autonomous and have the ability to move and actuate on their surroundings in an effort to provide services to users who may or may not be in physical proximity of the devices. These scenarios create business opportunities for organizations seeking to provide services to these users.

However, these opportunities create numerous questions that have not been adequately researched including applicable business models for these services, how value is co-created in these service settings, the role of network externalities, security and privacy issues, data analysis issues and big data techniques, and how mobile devices will make contact with other devices and form business relationships. In addition, very few empirical studies have been conducted on using the Internet of Things to provide such services.

The two papers accepted for the minitrack investigate these issues in different ways.

The first paper, titled "Toward Predicting Secure Environments for Wearable Devices" by Charles Walter, Ian Riley, Xinchi He, Ethan Robards and Rose Gamble identifies security issues in wearable technologies. In recent years, wearable devices have become more common for the average consumer. As devices need to operate with low power, many devices use simplified security measures to secure the data during transmission. While Bluetooth, the primary method of communication, includes certain security measures as part of the format, they are insufficient to fully secure the connection and the data transmitted. Users must be made aware of the potential security threats to the information communicated by the wearable, as well as be empowered and engaged to protect it. In this paper, the authors propose a method of identifying insecure environments through crowdsourced data, allowing wearable consumers to deploy an application on their base system (e.g., a smart phone) that alerts when in the presence of a security threat. The authors examine two different machine learning methods for classifying the environment and interacting with the users, as well as evaluating the potential uses for both algorithms.

The second paper, titled "Smart Cities: A Case Study in Waste Monitoring and Management" by André Castro Lundin, Ali Gurcan Ozkil and Jakob Schuldt-Jensen investigates smart waste management using case methodology. This paper explores the potential of employing sensor enabled solutions to improve on waste monitoring and collection in public trash bins. Through a user-centered design approach, an inexpensive monitoring system was developed and tested in pilot study. The system consists of wireless nodes that use ultrasonic sensors to measure the empty space in the bins, a sensor gateway that is based on Long Rage Wide Area Network (LoRaWAN) protocol and cloud-based back/front end for data collection, analysis and visualization. The system was evaluated through a pilot test, where six outdoor trash bins were remotely monitored at a university campus and a number of stakeholders were observed and interviewed. The results show that the existing technologies are mature enough to be able to develop and implement inexpensive add-on sensors to exiting trash bins, and employing such a system can provide the necessary insights to optimize waste collection processes, to avoid overfilled bins, and to improve the experience of the citizens.

We hope you enjoy the papers and their presentation at the conference. We thank the authors for submitting their work to make this another engaging minitrack. We also thank the reviewers for their valuable feedback. 